\title{
Fraternité, poste restante ? Projets d'écriture épistolaire en milieu scolaire
}

Brotherhood, poste restante? Epistolary writing projects in school

Sandrine Bazile, Pascale Dequin and Dominique Heches

\section{OpenEdition \\ Journals}

\section{Electronic version}

URL: http://journals.openedition.org/trema/5642

DOI: $10.4000 /$ trema.5642

ISSN: 2107-0997

Publisher

Faculté d'Éducation de l'université de Montpellier

\section{Electronic reference}

Sandrine Bazile, Pascale Dequin and Dominique Heches, «Fraternité, poste restante ? Projets d'écriture épistolaire en milieu scolaire », Tréma [Online], 53 | 2020, Online since 01 March 2020, connection on 03 March 2020. URL : http://journals.openedition.org/trema/5642 ; DOI : 10.4000/ trema.5642

This text was automatically generated on 3 March 2020.

Trema 


\section{Fraternité, poste restante ? Projets d'écriture épistolaire en milieu scolaire}

Brotherhood, poste restante? Epistolary writing projects in school

Sandrine Bazile, Pascale Dequin and Dominique Heches

\section{Introduction}

1 Dans le cadre du projet Éduquer à l'égalité dans la diversité, la Ligue de l'enseignement propose à des enfants et de jeunes adultes un travail sur les représentations, «en remettant à l'ordre du jour des valeurs de tolérance et de respect, qui sont constitutives du statut de citoyen responsable » (Jouons la carte de la fraternité, 2016). L'opération Jouons la carte de la fraternité se fonde sur une idée simple, celle de la bouteille à la mer:«le 21 mars de chaque année, à l'occasion de la Journée internationale pour l'élimination des discriminations, des enfants et des adolescents envoient des cartes postales à des anonymes tirés au hasard dans l'annuaire de leur département » (Ibidem).

2 En 2016, pour la deuxième année consécutive, cette opération a été menée dans deux types d'établissements auprès de publics d'âges et de situations variés, d'une part, des élèves de cours préparatoire et de cours élémentaire première année (dorénavant $\mathrm{CP}$ et CE1 $\left.1^{1}\right)$ d'une école élémentaire, d'autre part, des détenus du centre pénitentiaire de Perpignan, un public dit «contraint» de jeunes adultes hommes allophones issus de multiples horizons culturels et linguistiques. Faute de données collectées suffisantes pour la prison, nous ne rendrons compte ici que de l'expérience menée en milieu scolaire.

3 Dans le contexte tragique des actes terroristes qui ont touché la France en janvier et novembre 2015, dont nous savons qu'ils ont donné lieu à des réceptions diverses, et plus spécifiquement, dans le contexte de la réflexion autour de la fraternité en éducation et de l'éducation à la fraternité, ce projet nous semblait digne de 
considération, et ce, dans une double perspective: tout d'abord, ces expériences proposent une réflexion possible sur la façon dont l'école peut penser la notion même de fraternité en tant que valeur, dans sa dimension éthique ; ensuite, elles interrogent la manière dont cette éducation à la fraternité peut être menée en classe, au sein de pratiques langagières variées, orales et écrites, entre productions collectives, collaboratives et individuelles.

4 Après avoir rapidement présenté le projet tel qu'il a été mis en œuvre et a évolué, nous en préciserons l'arrière-plan théorique. L'analyse des données constituées de photographies des écrits produits, d'enregistrements d'échanges et d'entretiens semidirectifs collectées en 2016 se concentrera donc sur les productions de la classe de $\mathrm{CP}$ et CE1 et tentera de démêler la façon dont les pratiques langagières en classe permettent de faire émerger les représentations sur la fraternité et renseigne ses modalités de mise en actes dans une classe de CP/CE1. Il s'agira notamment d'interroger, au sein de ces pratiques, le statut de l'œuvre d'art mais surtout l'épaississement de la notion de fraternité au sein des productions successives (Chabanne \& Bucheton, 2002a ; 2000b). Nous tenterons ainsi de comprendre quelle définition de la fraternité se construit au sein des échanges et comment les élèves s'approprient cette notion, dans leurs écrits personnels.

\section{Penser la fraternité à l'aune des écrits et oraux réflexifs}

5 L'opération Jouons la carte de la fraternité est une " une opération de sensibilisation au respect et à la tolérance sur la base d'ateliers d'écriture et d'analyse de photographies " (Jouons la carte de la fraternité, 2016), proposée aux enseignants par la Ligue, qui vise «à déconstruire certaines représentations sur différents sujets de société » (Ibidem). Le projet présenté ici, repose sur un principe récurrent d'interactions entre pratiques langagières écrites et orales et sur un tressage permanent des productions. Par ailleurs, plusieurs disciplines y sont imbriquées (français, arts plastiques et enseignement moral et civique, dorénavant EMC).

\section{Un projet inséré dans un double cadre institutionnel et associatif}

\section{La place des valeurs dans les Programmes du cycle 2}

6 Depuis la parution des Programmes du cycle 2, on sait que pour l'école la transmission des valeurs et entre autres celle de la fraternité n'est plus une option mais une obligation, non pas en tant que connaissance théorique mais en tant que construction qui ne peut se réaliser que soutenue par une mise en œuvre efficace. Pour reprendre l'expression de Régis Debray, il convient de « ne pas laisser des générations naitre sous $\mathrm{X}$ en matière de valeurs " (Debray, 2009, p. 351), ce que rappellent les principes généraux de ces programmes : « la morale enseignée à l'école est une morale civique en lien étroit avec les principes et les valeurs de la citoyenneté républicaine et démocratique. Ces valeurs sont la liberté, l'égalité, la fraternité, la laïcité, la solidarité, l'esprit de justice, le respect et l'absence de toutes formes de discriminations. » (MEN, 2015a, p. 55)

7 À l'école, l'enseignement moral et civique refuse «l'imposition de dogmes ou de modèles de comportements » (Ibidem) mais vise plutôt à «l'acquisition d'une culture 
morale et civique et d'un esprit critique qui ont pour finalité le développement des dispositions permettant aux élèves de devenir progressivement conscients de leurs responsabilités dans leur vie personnelle et sociale " (Ibidem). En ce sens, les textes officiels préconisent l'articulation des valeurs, des savoirs et des pratiques, en sollicitant «le recours aux apprentissages et l'appropriation de savoirs (littéraires, scientifiques, historiques, juridiques)»(Ibidem, p. 56). Par ailleurs, l'enseignement moral et civique privilégie "la mise en activité des élèves» (Ibidem, p. 55) et leur « engagement» (Ibidem, p. 57) au sein de situations réelles aptes à favoriser leur formation "d'homme et de citoyen» (Ibidem), sans pour autant négliger le développement de leur « disposition à raisonner, à prendre en compte le point de vue de l'autre (...) au cours d'activités collectives et individuelles (discussion, argumentation, projets communs, coopération...)» (Ibidem, p. 56). Dans ces programmes, la notion de fraternité occupe une place assumée parmi les connaissances, capacités et attitudes visées : «Expliquer en mots simples la fraternité et la solidarité » (Ibidem, p. 61).

Parmi les méthodes et démarches proposées par les documents d'accompagnement du programme $2015^{2}$ (Méthodes et démarches, 2016), les débats figurent en bonne place (débat réglé, dilemmes moraux, discussion à visée philosophique, méthode de clarification de valeurs, conseils d'élèves, techniques des messages clairs) et sollicitent des pratiques langagières variées. Ainsi, si le programme de français, n'insiste pas, comme celui du cycle 3 , sur la valeur intrinsèque du texte littéraire ou de l'œuvre artistique en général pour mettre en scène et discuter des valeurs, des échos possibles et nombreux entre les pratiques de l'EMC et celles de la discipline français, principalement dans le cadre de pratiques discursives orales spécifiques (MEN, 2015a, p. 14). De la même façon, si les élèves de cycle 3 sont incités à investir personnellement l'œuvre en exprimant leur " jugement, à l'égard des personnages notamment » (MEN, 2015a, p. 109), le programme de français de cycle 2 n'évoque pas explicitement le même positionnement axiologique au regard du système de valeurs des élèves.

\section{Un projet initié par la Ligue de l'enseignement}

9 Le projet porté par la Ligue de l'enseignement veut faire réfléchir les élèves au concept de fraternité ; il ne s'agit nullement de conditionner ni de formater, ni non plus de penser à leur place mais bien au contraire de leur donner les moyens de construire le concept de fraternité « en s'affranchissant du conformisme moral » (Favey \& Coq, 2015) et en faisant émerger leurs représentations qui constitueront la matière première de cette réflexion. Par ce biais, on vise le développement de l'aptitude à l'autonomie morale et la prise de conscience d'une dichotomie entre valeurs et comportement. Ce constat n'est pas nouveau: déjà en 1985, Antoine Prost affirmait «que notre société [était] schizophrénique [...] On ne peut inculquer dans l'école et par l'école d'autres valeurs que celles de la société elle-même et il est vain d'espérer faire le contrepoids par l'école aux tendances d'une société. Entreprendre de restaurer dans l'école des valeurs dont on se gausse au dehors n'est pas rétablir l'équilibre : c'est exaspérer une contradiction » (Prost, 1985, p. 56).

La mise en œuvre est simple : une carte postale composée d'une photographie et d'un message de fraternité rédigé au cours d'ateliers d'écriture. Les destinataires sont invités à répondre à l'aide d'un coupon détachable à l'adresse de la Ligue de 
l'enseignement du département qui, sur le principe d'un service de poste restante, fait ensuite suivre les réponses aux jeunes expéditeurs, tout en conservant leur anonymat.

11 À chaque année correspond l'œuvre d'un photographe; en 2016, Pierre Faure est le lauréat ; il affirme dans le dossier pédagogique ${ }^{3}$ qui accompagne les photographies : « Je considère ma photo réussie lorsqu'elle est capable de dire des histoires différentes aux différentes personnes qui la regardent » (voir figure 1). L'affiche du projet Jouons la carte de la fraternité, 2016 reprend les six photographies intitulées respectivement: Préparatifs, Campement Rom, Les Tours, Graffiti, Marina et Le Refuge. Des vidéos ainsi que des légendes produites par le photographe lui-même et disponibles sur le site de la Ligue $^{4}$, reviennent sur le contexte de ces clichés et fournissent quelques pistes d'interprétation. Préparatifs qui met en scène deux sœurs en train de se coiffer avant une soirée d'anniversaire a été prise en 2012 sur le campement rom d'Ivry-sur-Seine. Campement Rom montre la précarité du quotidien d'une cinquantaine de familles originaires du nord-est de la Roumanie et installées sur un terrain vague d'Ile-deFrance en 2012. Marina est photographiée en 2015 dans le cadre d'un projet " précarité en milieu rural » : «elle vit dans une maison qui se délabre. Ses problèmes de santé vont bientôt l'obliger à quitter cette maison et ses animaux. » Le Refuge (2013) a été prise dans le centre d'hébergement d'urgence parisien du même nom : l'existence d'une telle structure qui accueille chaque jour près de 350 personnes sans domicile illustre, pour le photographe, "l'inefficacité de la gestion saisonnière du problème, sans politique sur le long terme.» Dans Les Tours (2010), sur laquelle on peut voir des immeubles du 13e arrondissement de Paris, l'artiste dit vouloir représenter « le béton » et «l'horizon bloqué ». Enfin, pour Graffiti (2010), Pierre Faure déclare avoir "passé plusieurs jours devant ce mur à attendre le "bon" passant»; ce qu'il apprécie sur le cliché, c'est «le contraste entre cette femme âgée et la violence du graff représentant des enfants guerriers ». 


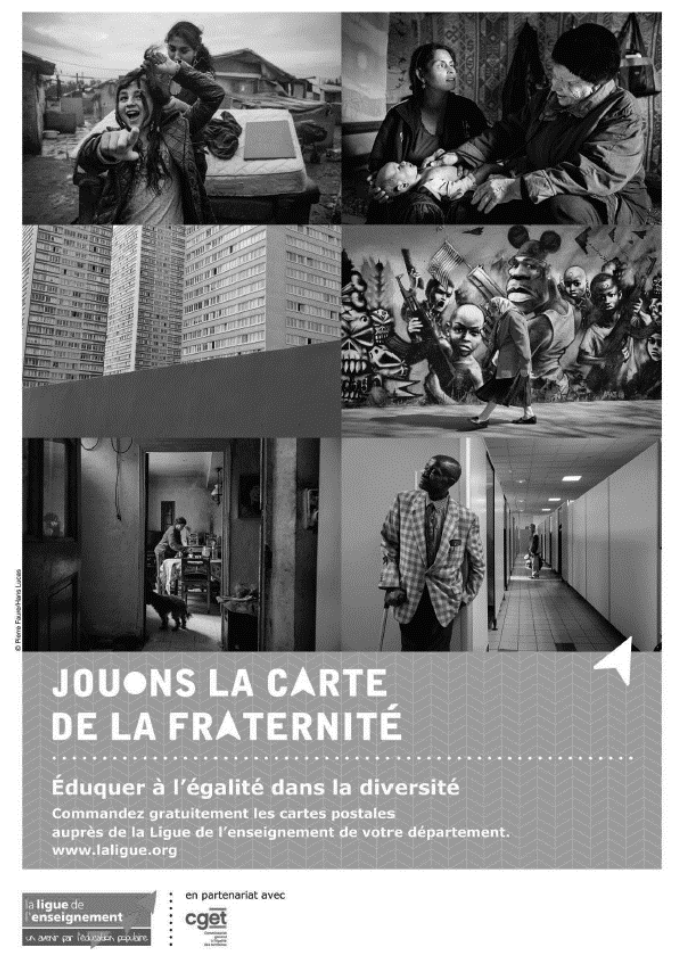

Figure 1 : Affiche du projet Jouons la carte de la fraternité, 2016 comprenant les photographies de gauche à droite et de haut en bas : Préparatifs, Campement Rom, Les Tours, Graffiti, Marina et Le Refuge.

12 Le dossier pédagogique qui accompagne l'opération contient des textes de référence témoignant de visions sur la fraternité en Europe, des liens vers des ressources en ligne, des lexiques thématiques ainsi que des séances-types à mettre en œuvre afin d'aider les enseignants et les éducateurs dans leur démarche. Ce dossier affiche des objectifs multiples qui ont partie liée avec les compétences du Socle commun de connaissances, de compétences et de culture (MEN, 2015b). Du point de vue de la formation de la personne et du citoyen, il s'agit «d'engager, avec les jeunes, une réflexion sur la diversité, les discriminations" mais également de favoriser une mise en acte constituée par l'interpellation d'un destinataire et sa réponse possible - « qui marque le début d'un engagement et peut déclencher une authentique prise de conscience, contribuant à l'apprentissage des valeurs de la fraternité : égalité, solidarité, justice, respect, etc.». En rapport avec le domaine 1, "Des langages pour penser et communiquer ", du Socle commun de connaissances, de compétences et de culture, il s'agit de "sensibiliser les jeunes à la lecture de l'image (...) dans la société contemporaine " (MEN, 2015b) de «développer l'esprit critique et l'imaginaire en confrontant idées, opinions et émotions » (Ibidem), objectifs auxquels participe la rédaction d'un «texte personnel» (Ibidem) réalisé en situation authentique de production et renvoyant à une pratique sociale de référence (Martinand, 1987 ; Reuter et alii, 2013).

\section{La mise en œuvre du projet}

13 La classe où se déroule le projet appartient à une école d'application de centre-ville, l'école Jules Ferry, située à Perpignan (Pyrénées Orientales). Le contexte socio-culturel 
de cet établissement est hétérogène, de même que le niveau de la classe double, composée d'un $\mathrm{CP}$ et d'un CE1. L'enseignante lance le projet début mars pour programmer un envoi le 21 mars. Après avoir explicité le calendrier du projet et ses phases successives - écrire une carte, choisir une adresse au hasard, un destinataire aléatoire, attendre une réponse éventuelle -, l'enseignante articule la première séance autour d'une " pluie d'idées » et d'un recueil de concepts et de représentations ; autour du mot "fraternité", chaque élève vient écrire au tableau numérique interactif (dorénavant TNI) un mot dont il est amené à justifier le choix. Les mots (voir figure 2) seront conservés pour les phases suivantes.

En 2015, l'enseignante avait travaillé autour de la notion de liberté après les attentats de janvier; au mois de mars, les représentations étaient encore présentes dans les mémoires et ont naturellement émergé les notions de valeurs républicaines autour des mots, liberté, égalité, fraternité. Dans un deuxième temps, la consigne est de produire en binôme un lexique autour des photos en trouvant des mots distincts de ceux déjà inscrits au TNI. Enfin, après une mise en commun, chaque binôme peut choisir la carte postale qui lui convient pour se consacrer à l'écriture. Ce travail, mené au brouillon avant la rédaction définitive, précède le choix d'un destinataire pris au hasard dans l'annuaire et la sortie au bureau de poste qui concrétise cet envoi, pensé comme une bouteille à la mer.

En mars 2016, la phase de planification est allégée et la mise en mots intervient plus rapidement; en outre, l'étape d'apport sur le lexique autour des photos est supprimée au profit du travail sur l'écriture épistolaire. Dans l'entretien semi-directif mené avec l'enseignante, celle-ci explicite ces choix qui participent d'une même intention; au lieu d'un apport lexical jugé «fastidieu[x] » et « inhibant » du point de vue de la parole des élèves, elle souhaite d'une part « fixer " plus nettement les contraintes génériques de la lettre, d'autre part privilégier l'investissement psychoaffectif des photographies: « je voulais qu'ils réagissent spontanément à ces photos, partir de leurs émotions premières ».

\section{Des soubassements théoriques implicites}

16 En tant que PEMF 5 , l'enseignante engagée dans cette action est rompue aux pratiques d'écriture ; référente culturelle de l'école d'application dans laquelle elle est en poste, elle est investie dans de nombreux projets qui articulent Éducation Artistique et Culturelle (dorénavant EAC) et EMC. Elle a été régulièrement impliquée dans des travaux de recherche et est donc initiée à certains concepts développés récemment en didactique du français. Sa pratique, bien qu'ordinaire, s'appuie sur un arrière-plan théorique que nous préciserons ultérieurement et qui témoigne d'une forme d'expertise.

Les travaux de Bakhtine sur la création verbale et la notion de genre fournissent ici un cadre théorique général. Le postulat sous-jacent à ces dispositifs, comme à cette recherche, est celui d'un «langage conçu comme "artefact culturel" et "instrument médiateur" de la pensée » (Crinon, 2002, p.123), à la fois lieu de « l'interaction sociale et de l'élaboration cognitive » (Ibidem), d'où sans doute l'intérêt de la didactique pour les pratiques langagières mises en œuvre « pour penser, pour négocier des significations et stabiliser des savoirs au sein des contextes scolaires " (Jaubert \& Rebière, 2002, p. 163). On parle et on écrit ici, pour paraphraser le titre de l'ouvrage de J.-Ch. Chabanne et D. 
Bucheton (2002a), - pour " penser, apprendre et se construire » en tant qu'homme et citoyen. Loin d'être réduit à des "fonctions de mémorisation ou de communication " (Ibidem, p. 2), le langage est envisagé pour ses «fonctions heuristiques» (Ibidem). Dans des disciplines diverses (français, arts plastiques et EMC), dans des contextes de productions différents, les sujets " élaborent progressivement les formes sémiotiques de leurs représentations, au cours de processus complexes de reprise-transformationassimilation" " (Ibidem p. 1). Ce faisant, au travers de ces pratiques langagières, il n'intègre pas seulement des apprentissages scolaires mais également des connaissances sur la langue et le discours (Crinon, 2002, p. 123).

Les milieux dans lesquels se sont déroulés les projets offrent de nombreuses situations d'échanges spontanés (remue-méninges, débats informels, échanges entre pairs en binômes), durant lesquelles, pour reprendre la terminologie bakhtinienne, se transmutent les "genres du discours premier " vers un "genre du discours second", ici la lettre. M. Jaubert et M. Rebière rappellent que cette transmutation permet de «mettre à distance, d'objectiver, de reconfigurer l'activité dans laquelle le locuteur est engagé, c'est-à-dire de l'arracher de son contexte in media res, pour la dire, la réorganiser, la restructurer, la représenter via des formes langagières conventionnelles, déposées dans la culture et partagées par la communauté dans laquelle il s'inscrit. » (2002, p. 166)

Ce sont ces qualités de "réflexivité comme distance à l'expérience " (Chabanne \& Bucheton, 2002a, p. 5) qui sont à l'œuvre au sein de ces deux projets et que favorisent la diversité des contextes de productions et la multiplication même des productions écrites et orales. Ces productions peuvent être qualifiées d' «intermédiaires ", au sens où J.-Ch. Chabanne et D. Bucheton ont défini ce terme, c'est-à-dire :

intermédiaires entre deux états d'un écrit à mettre en forme, entre deux états de pensée, entre les membres d'un groupe de travail, entre des écrits et des oraux, etc. On gardera deux sèmes essentiels: le caractère médiat, être une médiation entre deux sujets, entre deux discours, entre le sujet et lui-même ; le caractère transitoire et lié à des situations précises de travail (2002a, p. 20).

L'écrit et l'oral intermédiaires apparaissent, dans le cas de ces deux projets, comme des outils didactiques de la production écrite : le premier intérêt de ces formes d'écrits, qui "se situent entre le brouillon, promis à une destruction immédiate, et les formes dignes de conservation et d'évaluation" (Chabanne \& Bucheton, 2000, p. 23) et se caractérisent par la grande variété de leurs formes - «listes, écrits courts, schémas, dessins... » (Ibidem, p. 24) - est de considérer l'écriture comme processus et de prendre en compte ses effets en retour sur le scripteur, « mais aussi sur les gestes professionnels de l'enseignant en charge d'accompagner l'utilisation de ces écrits» (Bucheton, 2008). Le second intérêt est l'amplification du nombre et de la qualité des " échanges oraux observés autour de ces écrits » (Chabanne \& Bucheton, 2000, p. 23).

Si M. Jaubert et M. Rebière rappellent que cet oral réflexif est souvent « sous-estimé par [certains] enseignants » (2002, p. 168), en raison de sa labilité et son immédiateté, il nous semblait intéressant de décrire précisément les occasions où cet oral n'était pas majoritairement réservé « aux pratiques de transmission/vérification des savoirs ou de dialogue au service de l'instauration du conflit sociocognitif » (Ibidem) mais bien à l'élaboration d'un "point de vue nouveau, de nouveaux réseaux conceptuels et un nouveau contrôle de leur activité scolaire, dont sont tributaires les genres seconds » (Ibidem). 


\section{Protocole et analyse des données} séance de lancement, des photographies du TNI restituant le résultat des divers remueméninges (voir figures 2, 3 et 4), le collectage des productions écrites (voir figures 5, 6 et 7), ainsi que les entretiens semi-directifs menés auprès de l'enseignante et de six élèves choisis en fonction d'un profil précis selon leur degré d'implication dans le projet et leurs compétences (en termes de productions écrite et orale). Au cours de ces entretiens, il s'agira de faire expliciter à l'enseignante - en amont - ses intentions, ses objectifs prévisionnels et les motivations de ses choix, mais aussi - en aval - les réussites, échecs et écarts par rapport au prévisionnel ; pour les élèves, il s'agira de les faire revenir sur le sens qu'ils donnent au projet et sur leur définition personnelle de la fraternité.

Notre premier objectif est de préciser quelle définition de la fraternité est ici donnée et la façon dont cette définition se construit durant les échanges; pour cela nous nous intéresserons aux vidéos et aux photographies du TNI. Notre second objectif est de savoir si les élèves sont parvenus à s'approprier cette définition en analysant la façon dont elle est réinvestie dans leurs productions écrites mais également au fil des entretiens. Notre troisième objectif est de comprendre quelle expérience de la fraternité a été vécue par les élèves tout au long de ce projet.

Notre analyse qui emprunte à l'analyse de discours s'est efforcée de comprendre comment les différents états des productions langagières - de l'oral collectif à l'écrit en binômes et l'oral individuel) - ont pu se nourrir successivement et, ce faisant, épaissir la conception initiale que les élèves avaient de la fraternité.

Afin de mesurer ces progrès, nous nous sommes ainsi appuyées sur les «trois zones de problèmes » identifiées par J.-C. Chabanne et D. Bucheton (2002b, p. 28-29) : dans quelle mesure les scripteurs modifient-ils leur "position énonciative et pragmatique », au cours de l'opération de transmutation des écrits/oraux intermédiaires vers l'écriture de la carte postale? Du point de vue "sémantique et symbolique ", que révèlent les textes produits de l'investissement du concept de fraternité ? En lien avec les deux éléments précédents, quelles sont les traces d'assimilation «des codes linguistiques » spécifiques à l'écrit épistolaire et attestant cette appropriation?

\section{Quelle définition collective et individuelle de la fraternité ?}

\section{Un triple processus définitionnel}

La notion de fraternité est définie au cours des multiples échanges langagiers, essentiellement oraux et collectifs, menés par l'enseignante.

La première étape de ce processus définitionnel consiste en la présentation du projet ; cette étape occupe la première moitié de la séance. L'enseignante explique qu'il s'agit d'écrire à quelqu'un qu'on ne connait pas : « Nous allons écrire des messages autour de la fraternité et les envoyer à des gens qu'on ne connait pas, au hasard» (Vidéo 1-0005). Ce paradoxe ne manque pas de surprendre les élèves dont les commentaires sont éloquents : «C'est bizarre (Vidéo 1-0005), c'est étrange »; "normalement on écrit à quelqu'un qu'on connait» (Vidéo 2-0006), c'est différent». Pendant les premiers 
enregistrements, les élèves y reviennent à cinq reprises. Pour les élèves, la normalité consisterait à envoyer un courrier à des personnes déjà identifiées par les expéditeurs. Durant l'échange qui constitue la moitié du volume horaire de la séance, la préoccupation constante de l'enseignante semble être de régler ce paradoxe d'un destinataire anonyme et pourtant identifié. Pourtant, si la notion d'anonymat apparait ainsi à deux reprises dans le discours de l'enseignante, elle ne parvient pas à être formellement définie au cours des échanges qui révèlent même une sorte de malentendu sur son sens. D'un côté, les élèves cherchant à résoudre l'absurdité de cet acte, tentent de trouver une identité à ces destinataires «pris au hasard dans l'annuaire », « anonymes ", selon les propres termes de l'enseignante : «c'est comme si on écrivait à des mamans ou à des papas » (Vidéo 2-0006); ce faisant, ils cherchent à se reconnaitre, à reconnaitre l'être connu, en cet autre dont ils ne connaissent pas le nom. D'un autre côté, l'enseignante refuse l'anonymat de cette correspondance, insistant sur le fait «qu'on marquera son nom pour que les gens puissent nous répondre " (Vidéo 2-0006) ; ce que les élèves interprètent comme un simple geste de politesse.

Dans un second temps, la notion de fraternité se construit autour d'une activité de brainstorming (voir figure 2) complétée par une troisième et dernière phase réalisée lors des commentaires des images proposées à la description par l'enseignante.

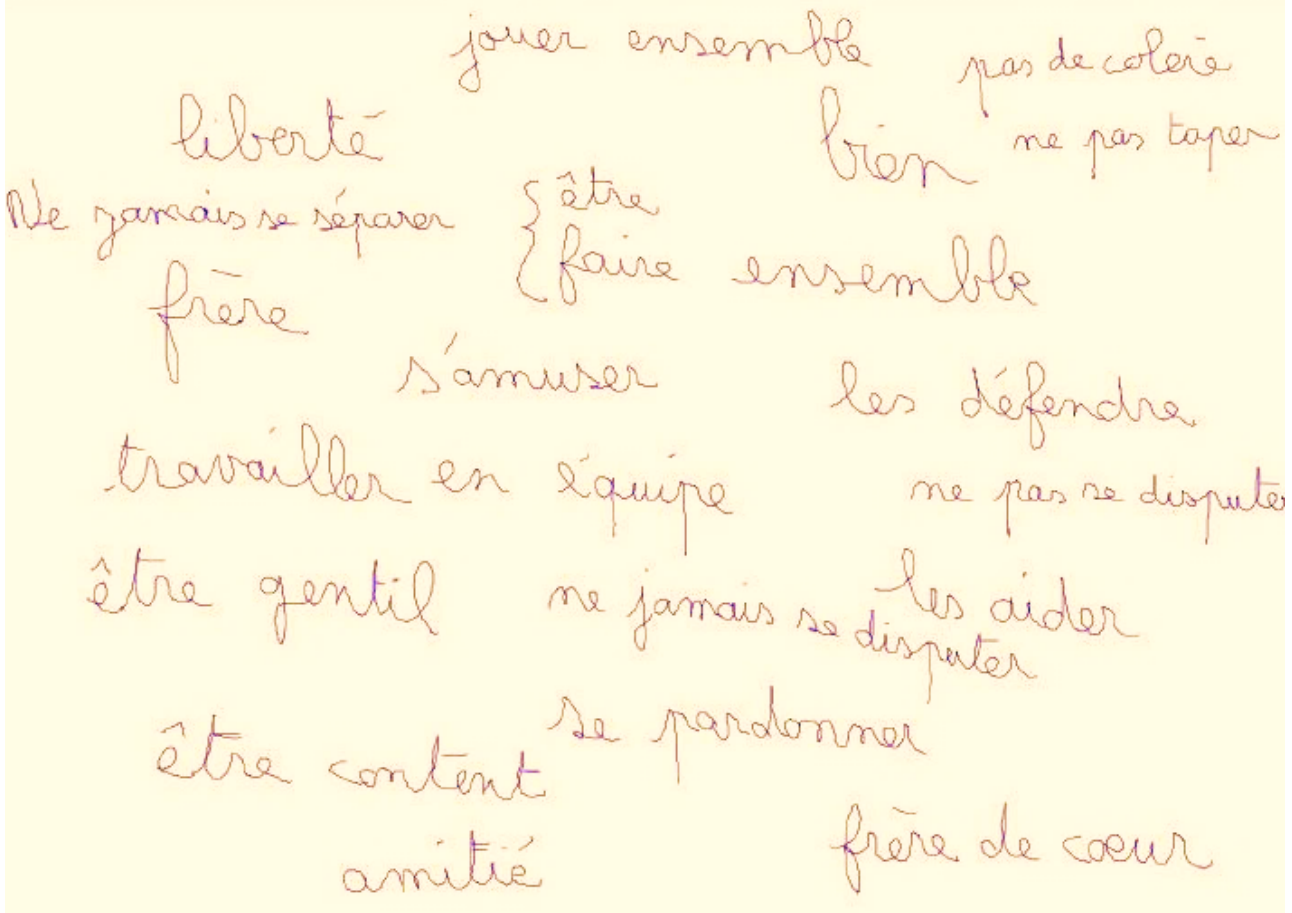

Figure 2 : Photographie du résultat du brainstorming réalisé dans la classe de CP/CE1

Lors de cette deuxième phase du processus définitionnel, la notion de fraternité se construit au travers de trois paradigmes qui apparaissent de façon récursive. Le premier concerne la dimension collective du terme: le mot «ensemble» revient à plusieurs reprises au cours de l'échange et il est mentionné deux fois au tableau, associé à des verbes d'action ("jouer», "être», "faire»); il est également associé à la collocation «travailler en équipe » ainsi qu'à l'expression «ne jamais se séparer ». Le deuxième paradigme concerne la dimension éthique de la notion de fraternité systématiquement associée dans le discours des élèves à la notion de gentillesse (« être 
gentil »; "bien »). Le troisième paradigme est celui du lien familial ou amical: les champs sémantique et lexical de la fraternité sont régulièrement utilisés dans le discours des élèves; le mot "frère » arrive ainsi assez tôt dans les échanges, employé seul et dans la collocation "frère de cœur" associé assez rapidement aux mots «famille », " ami », " amitié » auxquels vient répondre le champ lexical associé («ne jamais se séparer», "ne pas taper», «ne pas se disputer», "jouer», "se pardonner »...); l'intention sous-jacente est sûrement celle d'un lien pacifique entre les hommes; ce qu'A. Bidar définit comme une « amitié sociale » (2015, p. 3/10).

Les commentaires des photographies (voir figure 1), réalisées en classe entière, complètent ces paradigmes en partie seulement, notamment pour celui de la dimension collective : cela est particulièrement vrai pour les commentaires du cliché Campement Rom ("bébé s'occupe», "soigner») ou de Préparatifs (" ensemble», " partager l'argent ») qui se voient enrichis d'une dimension nouvelle, celle de la solidarité.

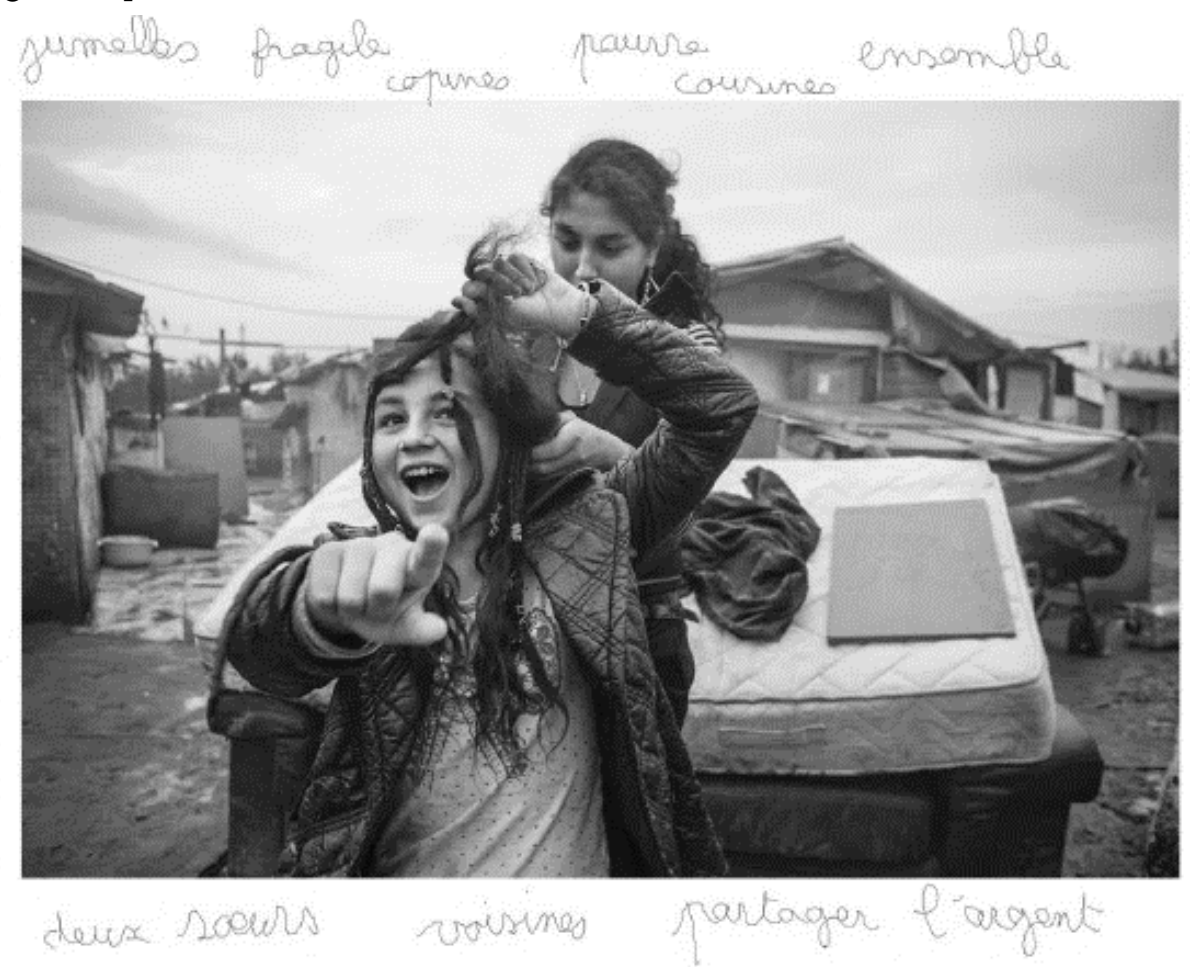

Figure 3 : Commentaire de la photographie Préparatifs

31 Les quatre autres photographies (Les Tours, Graffiti, Marina et Le Refuge) ne donnent lieu qu'à des descriptions du décor et des personnages sans lien apparent avec le thème de la fraternité, les échanges se limitant souvent à de simples mots ou à des tentatives pour rétablir un récit antérieur à la photographie selon le cas. Amenée à revenir sur ce point lors de l'entretien semi-directif, l'enseignante explique ce décalage de traitement par l'entrée qu'elle a choisie de privilégier : «ils ont réagi aux photos qui étaient les plus proches d'eux ». 


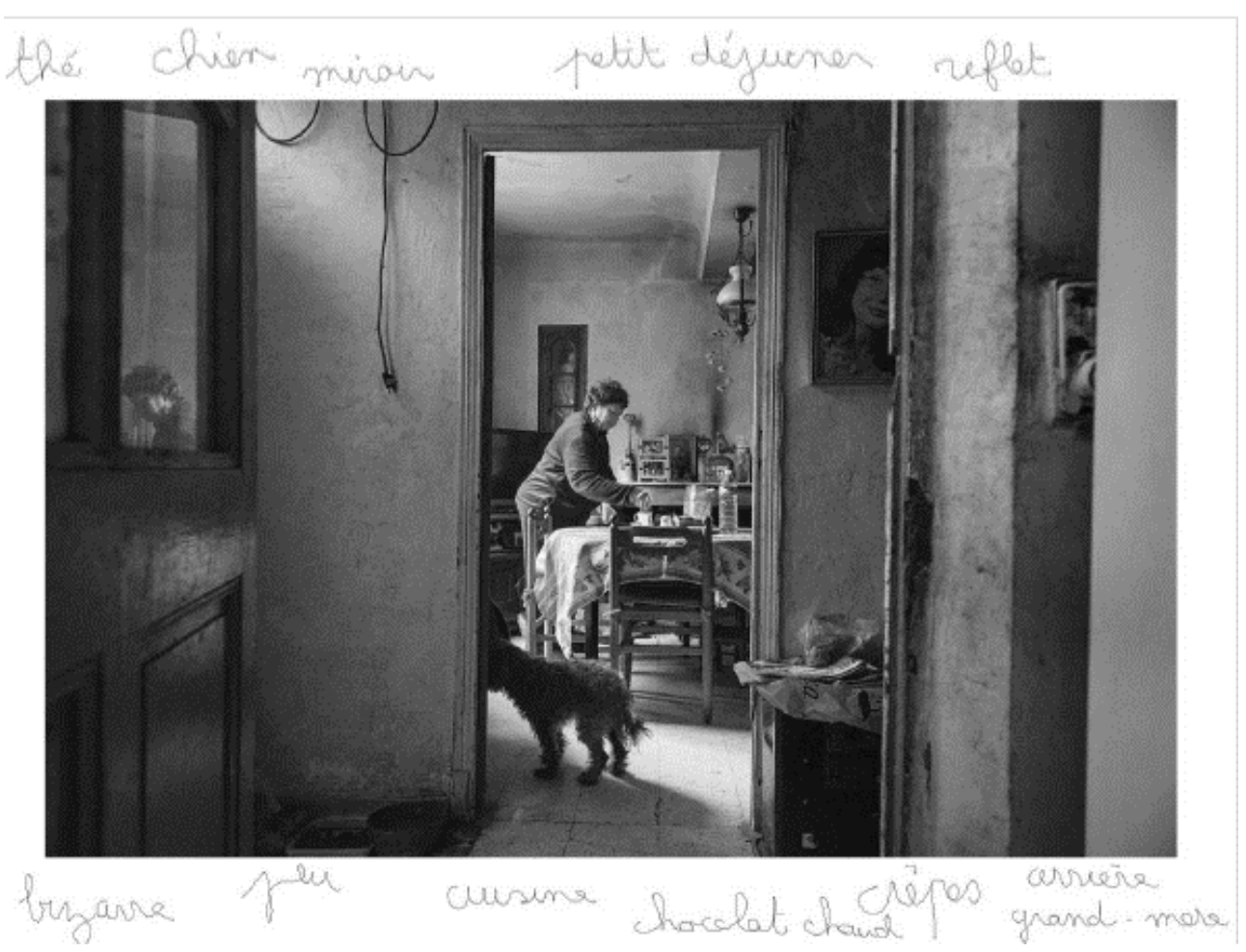

Figure 4 : Commentaire de la photographie Marina

\section{Une définition kaléidoscopique de la fraternité} " anonyme", "étranger " et «inconnu» ne parvient pas à être levée: elle est complexe, de l'avis de l'enseignante, pour des élèves de cet âge. Il nous semble que cette distinction pourrait être l'une des pistes pour interroger plus avant le principe même entre identité et altérité. En effet, si l'on s'en tient aux définitions proposées par le TLFI ${ }^{6}$, l'adjectif « anonyme ${ }^{7}$ » désigne celui « dont on ignore le nom, qu'on n'a pas pu identifier »; l'adjectif «inconnu ${ }^{8}$ » désigne "une personne dont on ignore le nom, l'identité, une personne étrangère (à un groupe faisant référence) dont on n'a pas (encore) fait la connaissance »; enfin, l'adjectif « étranger" " renvoie à " celui qui n'est pas familier à quelqu'un, qui n'a pas de relation avec lui, qui en est mal connu(e), distant(e)». ces définitions, pressent bien que la question du destinataire mérite pourtant d'être interrogée triplement car elle perçoit combien sa complexité est problématique pour les élèves; ces derniers soulignent en effet dans un premier temps le caractère anonyme de leur écriture: écrire à quelqu'un qu'on n'a pas pu identifier mais paradoxalement dont l'annuaire nous donnera le nom. Il s'agit dès lors d'écrire à quelqu'un d'inconnu, quelqu'un dont on n'a pas encore fait la connaissance. Mais le principe même de l'échange épistolaire vient réduire cette dimension en instaurant un principe de rencontre à distance avec l'inconnu qui vient réduire l'étrangeté même de ce correspondant plus tout à fait anonyme. «Je suis humain et rien de ce qui humain ne 
m'est étranger » écrivait Térence, cité ici par A. Bidar (2015, p. 92) l'anonyme, l'inconnu ne me sont pas étrangers puisqu'ils sont humains, puisqu'ils me ressemblent. Or les élèves, s'ils repèrent les signes évidents de ressemblance entre les êtres, lors de description de la photographie des deux sœurs, sont moins enclins à repérer à la fois ce qui distingue et ce qui rapproche ces êtres anonymes photographiés : la femme seule dans sa cuisine, l'homme noir handicapé, la vieille femme sur fond de tags urbains, les habitants absents de ces tours uniformes (voir figure 1). Sans doute la phase d'analyse des clichés - dont l'enseignante déplore, lors de l'entretien, la brièveté - n'a ainsi pas toujours permis de construire une lecture symbolique de ces images - difficile au demeurant pour des élèves de cet âge. De fait, la dimension universelle de la fraternité, cette « capacité à fraterniser au-delà du cercle restreint de [leur] proches » (Ibid. p. 86) : «Pour un réchauffement spirituel» (Ibid., p. 85) leur échappe probablement.

Avec le recul, lors de l'entretien, il apparait que l'identification des collectifs en présence, possible grâce à un travail de contextualisation des photographies (identification de la communauté Rom (figure 3), évocation de l'anonymat des tours de banlieue, explicitation des préparatifs de fête d'une communauté nomade...), aurait peut-être permis « d'éclaircir cette notion de rencontre avec la différence qui peut être comprise non seulement avec un autre singulier, et, par lui, avec le collectif qu'il représente, mais aussi avec d'autres communautés. " (Delamotte, François \& Porcher, 1997, p. 113) ; l'enseignante ajoute que les titres des photographies, ignorés à l'école, ou même l'intention de l'artiste auraient pu être un levier efficace en ce sens.

Par ailleurs, si le rapport d'inclusion politesse/solidarité est bien pressenti par les élèves, le lien entre politesse, respect, solidarité et fraternité ne peut être exploré plus avant probablement parce qu'il engage les élèves sur une réflexion plus conceptuelle que l'enseignante écarte car « trop exigeante en termes de temps».

Une autre dimension de la fraternité reste relativement en suspens pendant les échanges, celle de l'empathie. A. Bidar rappelle que la fraternité a partie liée avec le fait de «se soucier d'autrui, [de] se mettre à la place de l'autre " (Bidar, 2014, cité dans le dossier pédagogique, Ligue de l'enseignement, 2016); pourtant, peu de remarques d'élèves portent sur la question de l'identification à l'autre (à part au moment de la présentation du projet : on écrit à des personnes qui " pourraient être des mamans ou des papas »). Quelques rares commentaires - ceux des photographies Campement Rom (voir figure 1) représentant un bébé dans les bras d'une mère aveugle et Préparatifs (voir figure 3) - laissent entrevoir des signes d'empathie (sollicitude, compassion ou bienveillance) ; les écrits des élèves viendront sans doute nuancer cette remarque.

Les cooccurrences des modalisations et des négations durant le premier brainstorming indiquent que les notions des fraternité et de devoir sont régulièrement associées dans les propositions des élèves - la fraternité, c'est "ne jamais se séparer ", " ne pas taper ", «ne pas se disputer », «jouer", «se pardonner»- qui envisagent la notion de conflit comme incompatible avec celle de fraternité; or cette incompatibilité n'a pas pu être explorée, faute de temps : la fraternité exclut-elle le conflit, le désaccord ? Ou impliquet-elle un principe de résolution pacifique de celui-ci?

39 Si l'un des élèves cite le triptyque "Liberté égalité fraternité », aucun d'entre eux ne sait à quoi il renvoie exactement et c'est in fine l'enseignante qui explique qu'il s'agit «de la devise de la France, devise que l'on trouve sur les frontons des écoles et des mairies et qui parle des valeurs de la France " (Vidéo 3-0007): la devise résonne davantage comme un refrain connu qui ne fait pas véritablement sens pour les élèves. 
Seul Nawel parvient à conférer une valeur axiologique à la devise en précisant : « C'est le bien ». Pour les autres, la devise ressemble aux autres jeux d'homophonies ( «infinité »; « facteur ») auxquels se livrent les élèves autour du mot «fraternité ». La notion de fraternité est assez rapidement associée à celle de liberté («liberté d'expression " ", dit Paul) sans véritablement pouvoir expliquer pourquoi. Le principe d'égalité est étrangement absent des échanges, l'enseignante repoussant le moment où ce terme sera défini (" on en reparlera»). Le terme ne sera plus évoqué. Pourtant, à l'instar de François Dubet, force est de penser la solidarité comme condition de l'égalité :

La croyance en l'égalité fondamentale de tous est nécessaire, mais elle ne suffit pas à la recherche active de l'égalité sociale, qui repose sur des liens et des sentiments de solidarité, de ressemblance, de fraternité. Je peux désirer l'égalité sociale de ceux dont je me sens proche et relativement semblable, de ceux dont l'égalité me concerne et pour lesquels j'accepte de "payer » et de renoncer aux bénéfices des inégalités les plus grandes. Sans ces liens pratiques et imaginaires, la reconnaissance de l'égalité fondamentale, «les hommes naissent libres et égaux» n'engage pas nécessairement à rechercher une égalité réelle. (Dubet, 2014 : 39)

C'est l'enseignante qui donne la définition de la fraternité à la fin du remue-méninges «la fraternité c'est le fait d'agir ensemble comme des frères, même quand on ne se connait pas» (Vidéo 4-0008) -, définition n'est pas sans rappeler celle que donne A. Bidar : «La soif d'aimer le plus lointain comme son prochain » (2015, épilogue). À cette phase-là de la séquence, les élèves ne semblent pas faire véritablement le lien entre cette définition et le fait d'envoyer à une lettre quelqu'un que l'on ne connait pas comme si c'était un ami, il convient de vérifier si la suite, notamment la phase d'écriture, infléchit cette première analyse.

\section{Comment les élèves se sont-ils réappropriés les discours successifs sur la fraternité ?}

41 L'analyse des écrits permet de nuancer ces remarques et de mieux comprendre ce qui joue au moment de la phase de textualisation. Notre analyse concerne la dimension générique et discursive de ces productions et, à travers elle, les indices linguistiques qui confirment la prise en compte du genre épistolaire; elle s'intéresse également à la définition de la fraternité construite en filigrane; elle vise enfin l'analyse du rapport entre les images proposées aux élèves, les idées brassées dans la phase de brainstorming et dans les textes produits.

Du point de vue générique et discursif, on note la présence régulière de marqueurs énonciatifs employés dans une situation d'échange épistolaire ( cher monsieur, cher madame, ») ou d'indices attestant une production adressée (" bonjour », « au revoir »); on remarque en outre un écrit contextualisé : de façon presque systématique, la finalité de la carte est rappelée; il s'agit de souhaiter à son destinataire bonne fête de la fraternité. Ce point est d'ailleurs plus sensible au sein des écrits du projet 2016 par rapport au projet 2015 : l'enseignante signale avoir insisté sur la présence de ces codes épistolaires, même si elle a consacré moins de temps à la planification. En outre, les lettres hésitent entre différentes typologies textuelles : parfois descriptifs ou narratifs (voir figures 6 et 7), les textes sont plus rarement injonctifs (« Ne vous bagarrez pas!»), même si la quasi-totalité d'entre eux prennent la forme d'un dialogue à distance entre expéditeurs et destinataires - souvent dénué de tout lien avec la scène de la 
photographie - comme en témoigne la prédominance des marques des première et deuxième personnes du singulier dans les écrits de ces élèves.

Peu de productions font toutefois référence au fait que l'on écrit à une personne anonyme que l'on doit considérer comme son frère, même si certaines productions interrogent ce paradoxe d'une façon intéressante. Enzo et Ilyas écrivent par exemple : "je vous donne cette lettre parce que je vous aime de tout mon cœur»; Nayel et Mounir, encore: «j'espère que cette photo vous l'aimerez bien » ou encore Lylia et Isaure: "Je sais que tu ne me connais pas et je sais que je ne te connais pas. Peut-être qu'on s'est déjà vu dans la rue ", même si parfois ces occurrences sonnent de façon artificielle: "Je suis gentille avec vous je vous envoie cette photo parce que je vous aime bien" (Lylia et Isaure). Ces quelques occurrences figurent en tout cas, pour reprendre A. Bidar (2015), une tentative pour "mettre la personne au centre", pour "prendre en compte le fait de ces différentes rencontres", en tout cas donner corps à l'autre, lui conférer une réalité tangible, celle d'un être vivant avec lequel le partage est soudain rendu possible par l'acte d'écrire.

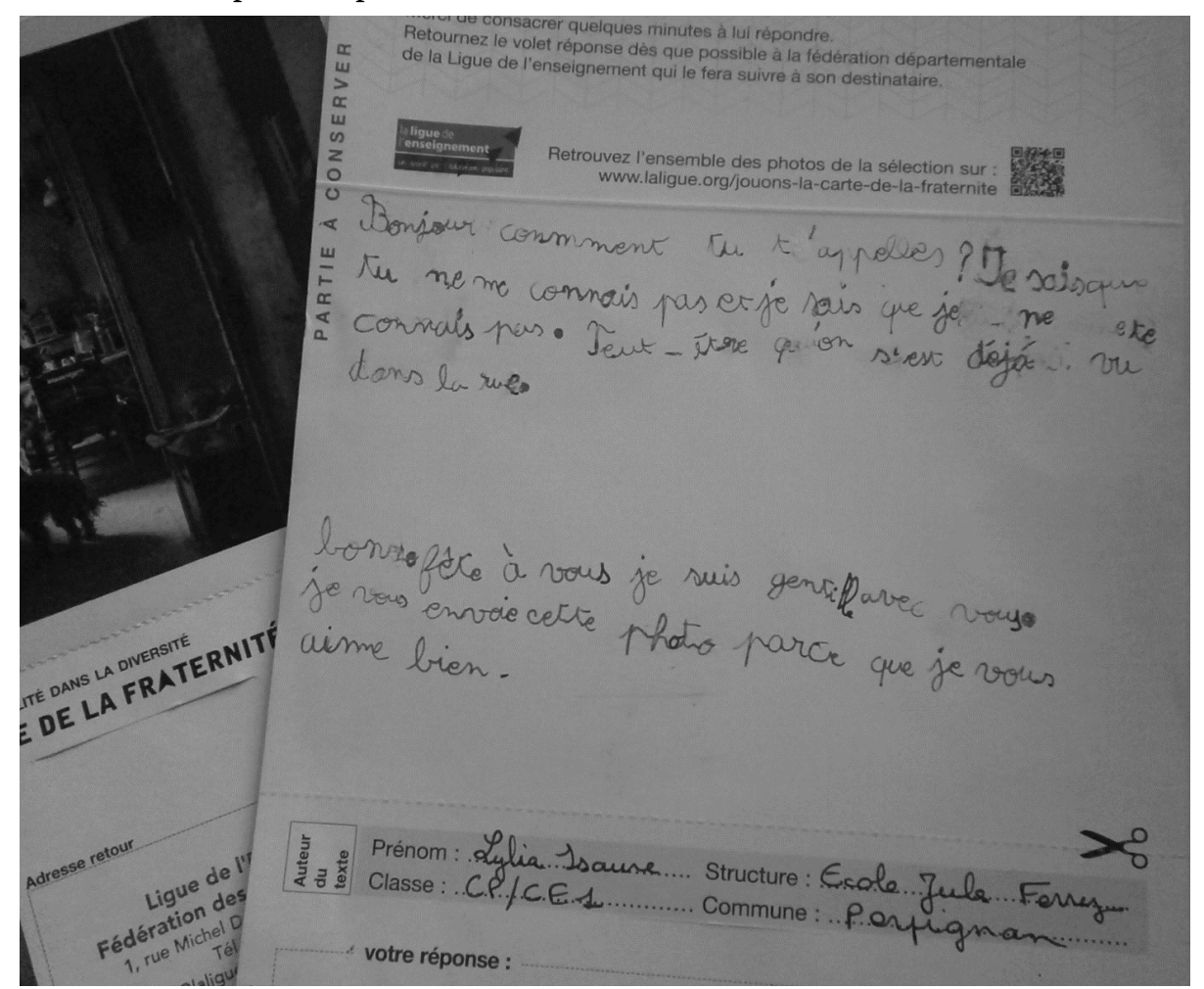

Figure 5 : Carte postale 1 (photographie : Marina)

$\mathrm{Du}$ point de vue sémantique et symbolique, les écrits témoignent d'une forme de réappropriation de la définition collective de la fraternité; nous trouvons ainsi des éléments très variés qui confirment pourtant la fragilité de la construction de cette notion - aucun des élèves interrogés lors des entretiens ne parviendra d'ailleurs à expliquer ce qu'est la fraternité. Tout d'abord, on relève une tentative isolée de définition: "la fraternité est comme un frère " (Mehdi, Younes). Ensuite, certaines injonctions traduisent un appel à une conduite pacifique, injonction qui apparait cohérente avec les propositions du remue-méninge initial. Affleure également parfois la dimension axiologique liée à la notion : « on dirait qu'il y a du bien parce que tout est calme» (Loubna et Marouane); cette dimension renverrait au fait, comme le rappelle 
Philippe Perrenoud, de "fonder la solidarité comme valeur et principe éthique » (2003).

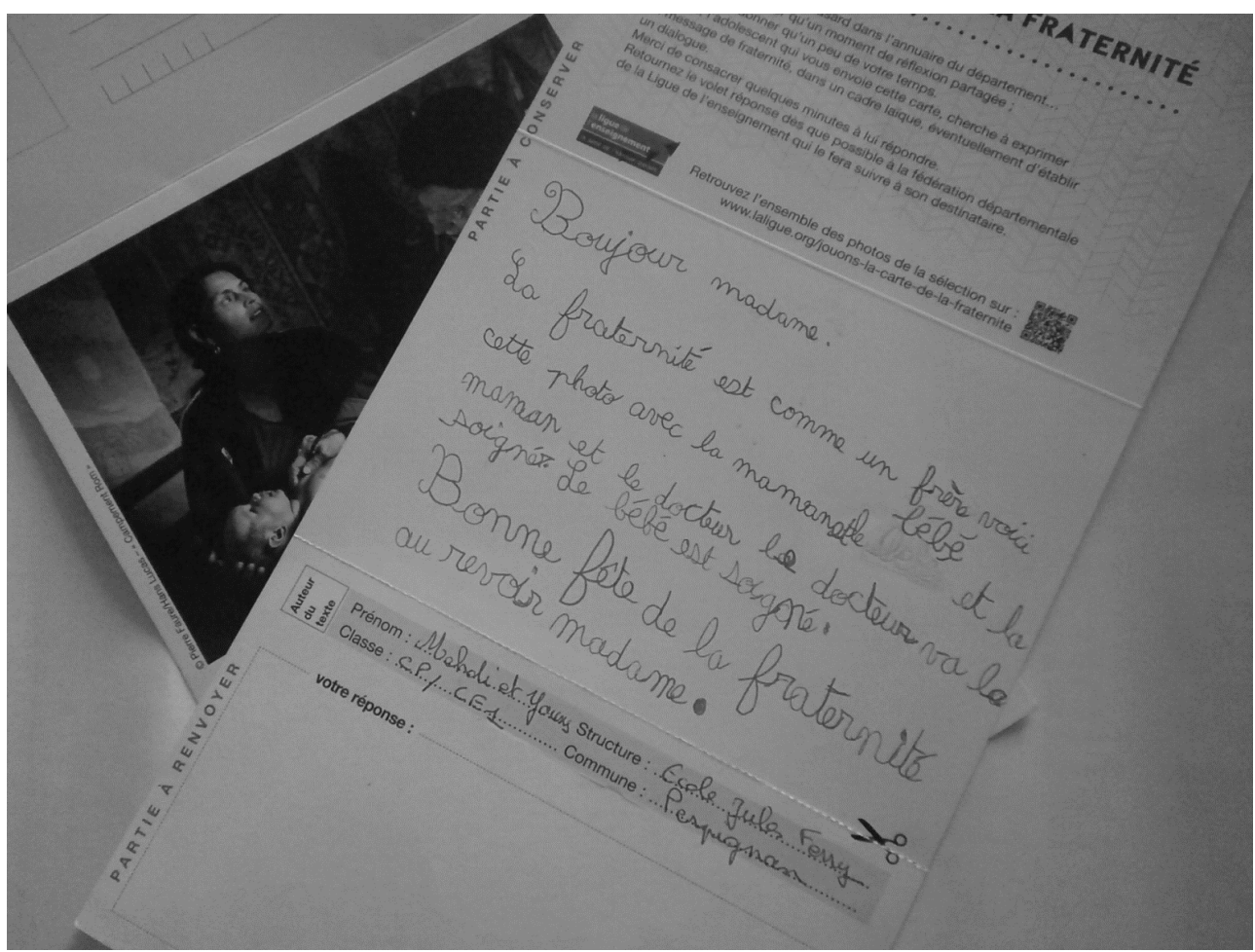

Figure 6 : Carte poste 2 (photographie : Campement Rom)

Enfin, une seule des cartes fait état, en filigrane, d'une certaine forme de compassion : "Oh qu'ils sont beaux ces enfants qu'ils sont mignons. Mais ils sont pauvres. Leur maison est cassée » (Soumeya et Clara). De la même façon, du point de vue du rapport à l'image, seules trois cartes font explicitement référence à l'image dont la description et l'interprétation auraient pu être un point de départ à la rencontre, à l'échange. Les élèves qui ont pris en compte la situation représentée sur les photographies, dans leurs écrits, l'ont fait parce qu'ils pouvaient les investir affectivement: ainsi seules les photographies Préparatifs et Campement Rom, sans doute parce qu'elles représentaient des situations proches de leur univers de référence et des personnages avec lesquels il était possible de s'identifier ont-elles donné lieu à des marques d'empathie. Pourtant lors des entretiens, comme dans les textes produits, il n'a pas été possible, pour ces élèves, de relier les émotions ressenties à la vue de ces clichés à un acte fraternel. 


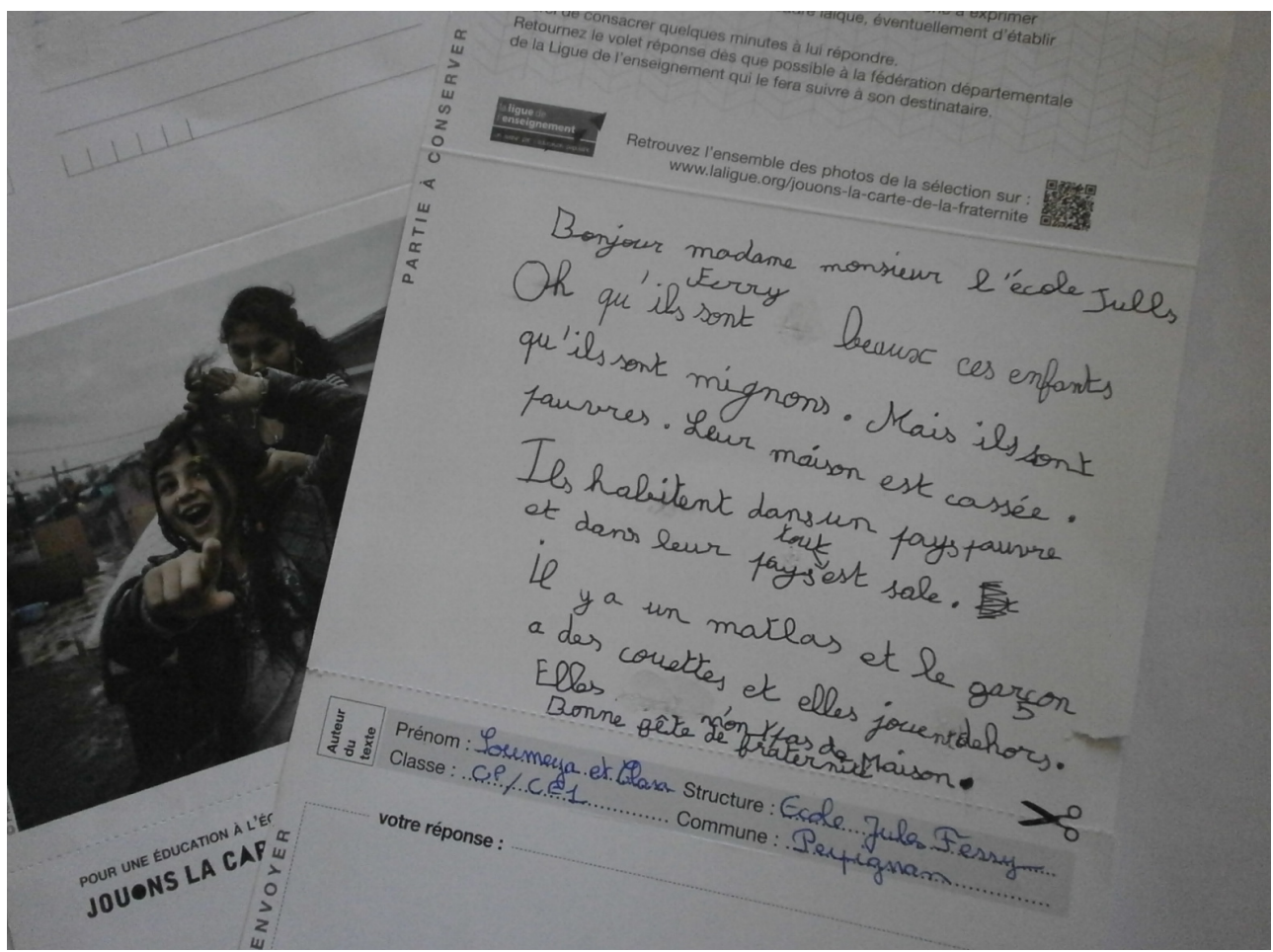

Figure 7 : Carte 3 (photographie Préparatifs)

\section{Conclusion : quelle expérience de la fraternité ?}

Deux processus parallèles semblent, selon nous, concourir à construire la construction de la notion de fraternité.

Le premier processus est le processus définitionnel enclenché par les temps d'échanges collectifs. L'analyse des données recueillies fait état de la difficulté des élèves à proposer une définition personnelle de la fraternité. La notion de fraternité ici construite interroge peu le rapport «liberté égalité fraternité ", encore moins le lien entre les peuples qui aurait pu être exploré, nous semble-t-il, lors de l'analyse d'image. En réduisant la phase d'apport lexical, l'enseignante souhaitait privilégier le rapport personnel des élèves aux photographies qui aurait pu constituer un départ tangible à une définition personnelle de la fraternité. Cependant, sans une contextualisation mieux assumée, une interprétation symbolique plus approfondie, ce temps de réactions personnelles sur l'œuvre court le risque de n'être ressenti par les élèves que comme un point de départ artificiel.

De fait, la notion de fraternité se centre sur une conception ordinaire du concept, sans doute plus accessible pour des élèves de CP/CE1 : la fraternité, c'est se sentir frère de quelqu'un que l'on ne connait pas, juste parce qu'on est humain.

49 Le second processus participe, lui, de l'expérience - Dubet parle d'ailleurs d'une éducation par l'expérience (2014, p. 94). C'est l'acte même de l'écriture de la fraternité qui fonde, de façon implicite, l'acte de fraternité. Pourtant, c'est précisément cette absence de réponse au courrier - ce sont sans doute là les limites du projet - qui fonde là encore la difficulté à établir un lien tangible entre les tentatives de définition données pendant les premières étapes du projet et l'expérience vécue de l'acte de fraternité et empêche, ce faisant, les élèves de s'approprier cette définition ou même de 
penser l'envoi de cette lettre comme un acte de fraternité, comme le font apparaitre les entretiens d'élèves. Interrogée sur le risque de ne considérer que la portée cosmétique d'une telle action, l'enseignante tient à réaffirmer la cohérence de son projet annuel qui cherche à articuler régulièrement EAC et EMC : ce n'est, selon elle, qu'au prix d'un travail « régulier et progressif », qui ne peut se limiter à l'investissement ponctuel dans un projet, que l'on peut réussir à faire vivre la fraternité au quotidien. Cette tâche de longue haleine mérite selon elle d'être pensée sur l'ensemble des trois cycles et dans une perspective interdisciplinaire ; selon nous, pour donner du sens à cette entreprise, il s'agirait également que l'histoire personnelle des élèves puisse faire écho à une dimension plus universelle de la fraternité.

Pour conclure, il nous semblait opportun de revenir sur une définition de la solidarité que donne François Dubet et qui semble faire écho à cette dernière remarque : « Pour le dire simplement nous sommes solidaires parce que nous adhérons à des mythes, à des récits, à des symboles qui nous constituent comme "frères" » (Dubet, 2014, p. 41). Au cours de ce projet, ces cartes envoyées à des anonymes, des inconnus réactualisent le principe d'une histoire commune; ces images racontent à chacun de nous une histoire dans laquelle chaque sujet peut se retrouver, se projeter et interroger l'autre à la fois dans sa ressemblance et sa différence... et admettre un lien réciproque avec l'autre, c'est peut-être ce qui manque aux lettres de ces écoliers pour qu'elles ne soient pas vouées à demeurer en " poste restante ".

\section{BIBLIOGRAPHY}

Bidar A. (2015). Plaidoyer pour la fraternité. Paris : Albin Michel.

Chabanne, J.-C. \& Bucheton, D. (2002a). Parler et écrire pour penser, apprendre et se construire : L'écrit et l'oral réflexifs (pp. 1-23). Paris : Presses Universitaires de France.

Chabanne, J.-C. \& Bucheton, D. (2002b). L'activité réflexive dans les écrits intermédiaires : quels indicateurs ? In J.-C. Chabanne et D. Bucheton (Dir.), Parler et écrire pour penser, apprendre et se construire: L'écrit et l'oral réflexifs (pp. 25-51). Paris : Presses Universitaires de France.

Chabanne, J.-C. \& Bucheton, D. (2000). Les écrits « intermédiaires ». La Lettre de l'Association DFLM, $\mathrm{n}^{\circ} 26,2000-1$, p. 23-27.

Crinon, J. (2002). Écrire le journal de ses apprentissages. In J.-C. Chabanne et D. Bucheton (Dir.), Parler et écrire pour penser, apprendre et se construire : L'écrit et l'oral réflexifs (pp. 123-143). Paris : Presses Universitaires de France.

Cohen-Azria C. (2013). Pratiques sociales de référence, In Dictionnaire des concepts fondamentaux des didactiques (pp. 175-178). Louvain-la-Neuve : De Boeck Supérieur.

Debray R. (2009). Le moment fraternité. Paris : Gallimard, 2009.

Delamotte-Legrand R., François F., Porcher L. (1997). Langage, éthique, éducation - Perspectives croisées. Rouen : Presses Universitaires de Rouen.

Dubet F. (2014). La préférence pour l'inégalité, comprendre les crises des solidarités. Paris : Seuil. 
Favey É. \& Coq G. (dir) (2015). Pour un enseignement laïque de la morale. Paris : Privat.

Jaubert, M. \& Rebière, M. (2002). Parler et débattre pour apprendre : comment caractériser un « oral réflexif » ?. In J.-C. Chabanne et D. Bucheton (Dir.), Parler et écrire pour penser, apprendre et se construire: L'écrit et l'oral réflexifs (pp. 163-186). Paris : Presses Universitaires de France.

Ligue de l'enseignement. (2016). Jouons la carte de la fraternité 2016, www.laligue.org/jouons-lacarte-de-la-fraternite/

Martinand, J.-L. (1987). Pratiques de référence, transposition didactique et savoirs professionnels en sciences et techniques. In Actes du Congrès de l'AIPELF.

Ministère de l'Éducation nationale. (2018). Méthodes et démarches. http://eduscol.education.fr/ pid34730-cid92404/methodes-et-demarches.html

Ministère de l'Éducation nationale (2015a), Programmes cycles 2, 3 et 4. http://

cache.media.education.gouv.fr/file/

MEN_SPE_11/67/3/2015_programmes_cycles234_4_12_ok_508673.pdf

Ministère de l'Éducation nationale (2015b), Socle commun de connaissances, de compétences et de culture, décret $\mathrm{n}^{\circ}$ 2015-372 du 31-3-2015 - J.O. du 2-4-2015 http://www.education.gouv.fr/ pid25535/bulletin_officiel.html?cid_bo $=87834$

Perrenoud, Ph. (2003). L'école est-elle encore le creuset de la démocratie ? Paris : Chronique sociale.

Prost A. (1985), Éloges des pédagogues. Paris : Seuil.

\section{NOTES}

1. Ces deux classes correspondent aux deux premières années de l'école élémentaire $\left(1^{\mathrm{e}}, 2^{\mathrm{e}}\right.$, Primaire), soit le début du cycle 2, cycle des apprentissages fondamentaux.

2. Notons que ces documents d'accompagnement ne sont plus accessibles en ligne.

3. Ce dossier n'est plus en ligne.

4. La page du projet 2016 n'est plus disponible en ligne.

5. Professeur des écoles maitre formateur

6. Article Fraternité accessible depuis http://www.cnrtl.fr/definition/fraternit\%C3\%A9

7. Article Anonyme accessible depuis http://www.cnrtl.fr/lexicographie/anonyme

8. Article Inconnu accessible depuis http://www.cnrtl.fr/lexicographie/inconnu

9. Article Étranger accessible depuis http://www.cnrtl.fr/lexicographie/etranger

\section{ABSTRACTS}

As part of the project Educating to equality in the diversity, the Teaching League proposes a job about representations, by bringing back to the front scene the values of tolerance and respect, which are part of the responsible citizen status. The operation Let's play the brotherhood card is based on a simple idea, the one of the message in a bottle : on the 21st of March of every year, during the International Day for the Elimination of Racial Discrimination, children and teenagers send some letters to anonymous people, randomly chosen in the phone book of their 
department. Each of these letters is made with a photo, resulting from an online pedagogic file, and a brotherhood's message written during a writing workshop. The recipients are invited to answer thanks to a detachable coupon to the address of the Teaching League of the department which forward them to the young senders.

The project is implemented in an elementary school, where the teacher seized the pedagogic file available online in order to initiate a triple work articulated around images reading, philosophic debate and writing production with pupils of first grade. The device, built on a common principle of weaving between individual, collaborative, and collective productions, articulate "intermediation writings" (Bucheton \& Chabanne, 2000 ; 2002a) and "reflexive orals" (Jaubert \& Rebière, 2002) to build a brotherhood in word and deed.

The analysis of the data made of photos and product writings, recordings about exchanges and semi directive interviews collected in 2016 will try to untangle the way language practices in class can be a training ground for brotherhood by questioning the status of masterpiece - here photography - but especially the thickening of the notion of brotherhood such pupils build it inside successive productions (Chabanne \& Bucheton, 2002a ; 2002b).

Dans le cadre du projet Éduquer à l'égalité dans la diversité, la Ligue de l'enseignement propose un travail sur les représentations, en remettant à l'ordre du jour des valeurs de tolérance et de respect, qui sont constitutives du statut de citoyen responsable. L'opération Jouons la carte de la fraternité se fonde sur une idée simple, celle de la bouteille à la mer : le 21 mars de chaque année, à l'occasion de la Journée internationale pour l'élimination des discriminations, des enfants et des adolescents envoient des cartes postales à des anonymes tirés au hasard dans l'annuaire de leur département. Chacune de ces cartes est composée d'une photographie, issue d'un dossier pédagogique fourni en ligne, et d'un message de fraternité rédigé au cours d'ateliers d'écriture. Les destinataires sont invités à répondre à l'aide d'un coupon détachable à l'adresse de la Ligue de l'enseignement du département qui les fait ensuite suivre aux jeunes expéditeurs.

Au sein d'une école élémentaire de Perpignan où le projet est mis en œuvre, une enseignante formatrice s'est emparée du dossier pédagogique disponible en ligne pour amorcer, avec ses élèves de cours préparatoire et de cours élémentaire première année, un triple travail articulé autour de la lecture d'images, du débat philosophique et de la production d'écrit. Le dispositif, construit sur un principe commun de tissage entre productions individuelles, collaboratives et collectives, articulent « écrits intermédiaires » (Bucheton \& Chabanne, 2000 ; 2002a) et « oraux réflexifs» (Jaubert \& Rebière, 2002) pour tenter de construire une fraternité en actes et en paroles.

L'analyse des données constituées de photographies des écrits produits, d'enregistrements d'échanges et d'entretiens semi-directifs collectées en 2016 tentera de démêler la façon dont les pratiques langagières en classe peuvent être un lieu de formation à la fraternité notamment en interrogeant le statut de l'œuvre d'art - ici la photographie - mais surtout l'enrichissement de la notion telle que les élèves la construisent au sein des productions successives (Chabanne \& Bucheton, 2002a; 2002b).

\section{INDEX}

Keywords: brotherhood, intermediate writings/orals, epistolary writing, photography, elementary school, first grade

Mots-clés: fraternité, écriture épistolaire, écrits/oraux intermédiaires, photographies, école élémentaire, cycle 2 
AUTHORS

SANDRINE BAZILE

Université de Montpellier, Faculté d'Education, LIRDEF

PASCALE DEQUIN

Université de Montpellier, Faculté d'Education

DOMINIQUE HECHES

Université de Montpellier, Faculté d'Education 